\title{
Apoptotic induction induces Leishmania aethiopica and L. mexicana spreading in terminally differentiated THP-1 cells
}

\author{
RAJEEV RAI, PAUL DYER, SIMON RICHARDSON, LAURENCE HARBIGE and \\ GIULIA GETTI* \\ Department of Life and Sport Science, University of Greenwich, Chatham Maritime, Kent, ME4 4TB, UK
}

(Received 11 April 2017; revised 24 May 2017; accepted 25 fune 2017)

\begin{abstract}
SUMMARY
Leishmaniasis develops after parasites establish themselves as amastigotes inside mammalian cells and start replicating. As relatively few parasites survive the innate immune defence, intracellular amastigotes spreading towards uninfected cells is instrumental to disease progression. Nevertheless the mechanism of Leishmania dissemination remains unclear, mostly due to the lack of a reliable model of infection spreading. Here, an in vitro model representing the dissemination of Leishmania amastigotes between human macrophages has been developed. Differentiated THP-1 macrophages were infected with GFP expressing Leishmania aethiopica and Leishmania mexicana. The percentage of infected cells was enriched via camptothecin treatment to achieve $64 \cdot 1 \pm 3 \%$ (L. aethiopica) and $92 \pm 1 \cdot 2 \%$ (L. mexicana) at $72 \mathrm{~h}$, compared to $35 \pm 4 \cdot 2 \%$ (L. aethiopica) and $36 \cdot 2 \pm 2 \cdot 4 \%$ (L. mexicana) in untreated population. Infected cells were co-cultured with a newly differentiated population of THP-1 macrophages. Spreading was detected after $12 \mathrm{~h}$ of co-culture. Live cell imaging showed inter-cellular extrusion of L. aethiopica and L. mexicana to recipient cells took place independently of host cell lysis. Establishment of secondary infection from Leishmania infected cells provided an insight into the cellular phenomena of parasite movement between human macrophages. Moreover, it supports further investigation into the molecular mechanisms of parasites spreading, which forms the basis of disease development.
\end{abstract}

Key words: In vitro model, spreading, Leishmania aethiopica, Leishmania mexicana, human monocytes, infection, THP-1, Retinoic acid.

\section{INTRODUCTION}

Unicellular protozoan parasites belonging to the genus Leishmania are the causative agent of a spectrum of human diseases known as leishmaniasis. Over 350 million people worldwide are at risk of contracting the disease which is endemic in 98 countries (Alvar et al. 2012). Moreover, current longterm conflicts and consequent migration of infected individuals from endemic areas has increased not only the number of reported cases but also the risk of an increase in the number of countries becoming endemic of the disease (Kedzierski, 2010; Stamm, 2016). Depending on the species of parasite and host genetic background, disease manifestation ranges from asymptomatic to cutaneous skin ulceration to life threatening infection of liver and spleen, leading to death, if untreated. No vaccine is currently available and effectiveness of existent drugs is limited due to toxicity and emergence of resistant parasites, making research on disease development and host-pathogen interaction a major priority in public health. The transmission of leishmaniasis is initiated when the sandfly injects the parasite

* Corresponding author: Department of Life and Sport Science, University of Greenwich, Chatham Maritime, Kent, ME4 4TB, UK. E-mail: G.T.M.Getti@greenwich.ac.uk infective stage (metacyclic promastigotes) during a blood meal. The total number of promastigotes inoculated is low (100-1000) (Rogers et al. 2004; Kimblin et al. 2008) with approximately $90 \%$ killed instantaneously by the host complement system despite the protective action of the sandfly saliva (Dominguez et al. 2003; Gomez and Oliveira, 2012). Disease can only develop in those cases where the surviving parasites successfully establish themselves within the definitive host cells, the macrophages.

Survival inside host macrophages depends on the ability of well characterized virulence factors (e.g. GIPLs, GP63, LPG, PPG and KMP-11) to inhibit macrophages anti-microbial activity (Bifeld and Clos, 2015). Leishmania inhibits multiple host pathways including lysosomal fusion (Lodge and Descoteaux, 2005), inflammatory cytokine and chemokine production (Cameron et al. 2004; Gregory et al. 2008), and MHC class II expression (De Souza et al. 1995). These virulent functions provide sufficient time for promastigotes to successfully transform into amastigotes, which establish themselves within the parasitophorous vacuole and start to actively replicate. Eventually intracellular parasites are released and spread to uninfected cells. In mice models, a silent phase, lasting 4-5 weeks was identified whereby the 
amplification of parasites in the dermis takes place without the formation of a lesion. The development of a lesion coincides with the killing of the parasites (Belkaid et al. 2000). Although the cellular and molecular mechanism behind Leishmania spread remains largely unknown, the above findings indicate a silent spreading takes place. The prime reason for this slow advancement is the lack of an effective in vitro model to represent this stage of host-parasite interaction. It has been widely assumed that Leishmania could egress via host cell lysis as previously observed for numerous human pathogens such as Shigella flexneri and Listeria monocytogenes (Ashida et al. 2011). Nevertheless, multidimensional live cell imaging has shown evidence that spreading of $L$. (L). amazonensis amastigotes between mice macrophages can also take place via a regulated mechanism resembling apoptosis (Real et al. 2014). Interestingly the existence of this mechanism is species-specific with no evidence of apoptotic induction detected during $L$. $(V)$. guyanensis (DaMata et al.2015). However, no data are available for other Leishmania species or, more importantly, on whether a similar mechanism exists in human macrophages. The choice of host cell to support Leishmania infection makes significant differences to the parasites susceptibility to drugs (Seifert et al. 2010) and it cannot be excluded that it would also impact on the parasites behaviour during spreading. Studies carried out directly on human cells are needed but are complicated by the fact that available models allow study of infection over a relatively short period of times $(72 \mathrm{~h}$ maximum), which is generally insufficient for spreading of amastigotes to take place (Getti et al. 2008).

This study aimed to develop an in vitro model, which allowed the study of long-term Leishmania infection and dissemination in human macrophages. This was successfully achieved by employing a cell-to-cell infection strategy whereby enriched populations of Leishmania aethiopica and Leishmania mexicana infected cells were used to infect uninfected recipient macrophages. The percentage of infected cells in the mixed population doubled within $12 \mathrm{~h}$ from co-culture without significantly affecting viable cell number, suggesting infection spreading was taking place. Live microscopy confirmed parasite movement between infected and recipient cells and gave an initial insight into the process of Leishmania extrusion.

MATERIALS AND METHODS

\section{Parasite culture}

Previously described promastigotes of Leishmania aethiopica (MHOM/ET/72/L100) and Leishmania mexicana (MNYC/BZ/62/M379) constitutively expressing GFP were used in this study (Patel et al. 2014). The reporter gene, GFP, was integrated in the genome, downstream of the promoter of the
$18 \mathrm{~S}$ rRNA. The presence of several copies of the $18 \mathrm{~S}$ rRNA gene tandemly arrayed in the Leishmania parasite genome is advantageous in that introduction of transgenes into the chromosome has no detrimental effect on parasite survival (Mißlitz et al. 2000).

For simplicity, GFP expressing clones were named as: L8G for L. aethiopica and M5G for L. mexicana. The promastigotes were grown in Schneider's Drosophila Medium (Thermo Fischer Scientific, UK) supplemented with 23\% Foetal Calf Serum (FCS) (Thermo Fischer Scientific) and $1 \times$ Penicillin-Streptomycin-Glutamine (Thermo Fischer Scientific) at neutral $\mathrm{pH}$. The parasites were sub-cultured once a week at a concentration of $1 \times 10^{6} \mathrm{~mL}^{-1}$, supplemented with $700 \mu \mathrm{g} \mathrm{mL}$ of G418 (Sigma-Aldrich, UK) and were incubated at $24{ }^{\circ} \mathrm{C}$ cooling incubator.

\section{Human cell culture}

Human THP-1 monocytes (ATCC, TIB-202) were sub-cultured every 3 days at a concentration of $2.5 \times 10^{5} \mathrm{~mL}^{-1}$ in complete RPMI 1640 medium ('Thermo Fischer Scientific) and incubated at $37^{\circ} \mathrm{C}$ in a humidified $5 \% \mathrm{CO}_{2}$ incubator. The medium was made complete following supplementation with $10 \%$ FCS and $1 \times$ Penicillin-Streptomycin-Glutamine.

\section{Infection of differentiated THP-1 macrophages with GFP expressing Leishmania}

Experimental infection with $L$. aethiopica and L. mexicana was carried out as previously described (Patel et al. 2014). Briefly, peanut lectin agglutination was used to isolate the metacyclic promastigotes from stationary phase cultures. This infectious form of the parasite was used to infect differentiated THP-1 macrophages at a ratio of 10:1 (parasites to cell ratio). This differentiation was achieved by treating THP-1 monocytes with $1 \mu \mathrm{M}$ of retinoic acid (Sigma-Aldrich) for $72 \mathrm{~h}$ prior to infection (Ogunkolade et al. 1990). The infected cells was seeded on a 24 well culture plate and incubated at $37^{\circ} \mathrm{C}$ in a humidified $5 \% \mathrm{CO}_{2}$ incubator. The percentage of infection for L. aethiopica and L. mexicana was determined through flow cytometry (BD Bioscience, UK) at 24, 48, 72 and $96 \mathrm{~h}$.

\section{Induction of apoptosis}

Apoptotic induction was carried out by treatment with $3 \mu \mathrm{M}$ of camptothecin (Sigma-Aldrich) at $0 \mathrm{~h}$ after infection. Excess camptothecin from $L$. mexicana and L. aethiopica infected cells were washed away with $1 \times$ PBS after 5 and $24 \mathrm{~h}$ after infection, respectively. Following the completion of washing step, the infected cells were re-suspended in 
complete RPMI medium and incubated at $37^{\circ} \mathrm{C}$ in a humidified $5 \% \mathrm{CO}_{2}$ incubator for $72 \mathrm{~h}$.

\section{Detection of cellular apoptosis}

PE Annexin V assay kit I (BD Biosciences) was used to determine apoptosis. The assay is based on Annexin $\mathrm{V}$ protein binding onto the externalized phosphatidyl serine (PS), which is an early marker for apoptotic cells. The kit also contains 7-AAD dye, which intercalates with DNA if the cell membrane is damaged, hence measuring necrosis. Briefly, the harvested cells were centrifuged at $500 \boldsymbol{g}$ for 10 min and washed twice with ice-cold $1 \times$ PBS. After re-suspension in $1 \times$ binding buffer at a cell concentration of $1 \times 10^{6} \mathrm{~mL}^{-1}, 100 \mu \mathrm{L}$ of cells were then stained with $5 \mu \mathrm{L}$ of PE Annexin $\mathrm{V}$ and $5 \mu \mathrm{L}$ of $7 \mathrm{AAD}$ and incubated for $15 \mathrm{~min}$ in the dark at room temperature. Finally, $400 \mu \mathrm{L}$ of $1 \times$ binding buffer was added to the stained cells and the percentage of Annexin $\mathrm{V}$ positive/ 7AAD negative (apoptosis) cells were analysed through flow cytometry within $1 \mathrm{~h}$ of staining.

\section{Infection of differentiated THP-1 macrophages with Leishmania infected cells}

After $72 \mathrm{~h}$ incubation of camptothecin treated L. aethiopica and L. mexicana infected cells, the concentration of viable cells was determined via trypan blue stain (Sigma-Aldrich). The infected cells were then centrifuged at $500 \mathrm{~g}$ for $10 \mathrm{~min}$ and the supernatant removed before co-culturing with a freshly prepared batch of differentiated THP-1 macrophages. Three different infection ratios were tested including $10: 1,5: 1$ and $1: 1$ (infected cell per differentiated THP-1 macrophage ratio). The percentage of infection was determined after $0,12,24$ and $48 \mathrm{~h}$ of co-culture through flow cytometry. For this experiment, $72 \mathrm{~h}$ incubated non-treated L. aethiopica and L. mexicana infected cells co-cultured with differentiated THP-1 macrophages were taken as a negative control.

\section{Fluorescent microscopy}

THP-1 macrophage from 48 and $96 \mathrm{~h}$ incubation were centrifuged at $500 \boldsymbol{g}$ for $5 \mathrm{~min}$ before washing twice with $1 \times$ PBS. Equal volume $(20 \mu \mathrm{L})$ of suspended cells was added onto microscopic slides before applying a drop of Prolong gold anti-fade mount with diamidino-2-phenylindole (DAPI) (Thermo Fisher Scientific). The resulting samples were then visualized through a fluorescent microscope (Nikon, UK).

\section{Real time lapse microscopy}

A fresh population of differentiated THP-1 macrophages at a concentration of $1 \times 10^{5} \mathrm{~mL}^{-1}$ was labelled with $0.5 \times$ CellMask $^{\mathrm{TM}}$ orange plasma membrane stain (Thermo Fischer Scientific) for $15 \mathrm{~min}$ at $37^{\circ} \mathrm{C}$. After washing three times with $1 \times \mathrm{PBS}$, the stained cells were re-suspended in a previously described cell imaging buffer solution (Richardson et al. 2008). After seeding in a 6 well culture plate containing $1 \times 10^{5}$ of apoptotic induced L. aethiopica and/or L. mexicana infected cells, the co-culture sample was then mounted on $37^{\circ} \mathrm{C}$ heated stage connected to a fluorescent microscope. Using an in built digital camera (Nikon), live images were acquired every $5 \mathrm{~min}$ with bright field and TRITC filters for a maximum period of $12 \mathrm{~h}$ under $20 \times$ objectives (Nikon). After acquisition, images were processed by NIS Elements version 4.2 software, which allowed for the construction of multidimensional time-lapse videos.

\section{Flow cytometry}

To determine the percentage of infection, the infected cells were extensively washed with $1 \times$ PBS before analysis with flow cytometry. Following an initial gating with Forward scatter (FSC) and Side scatter (SSC) parameters, the THP-1 population was further gated using a histogram plot to separate GFP positive (infected cell) from GFP negative (uninfected cell) population.

\section{Statistical analysis}

Data shown are representative of at least three independent experiments performed in three biological replicates. Data represents mean values with statistical error mean (S.E.M.). Statistical analysis was performed using student $t$ test provided by GraphPad Prism (CA, USA) software and $P$ values less than $0.05(P<0.05)$ were considered significant differences between two independent samples.

\section{RESULTS}

\section{Infection of THP-1 macrophage with Leishmania promastigotes}

Current in vitro models of Leishmania infection in human cells are based on promastigotes infection of macrophage cell lines such as THP-1 (Ogunkolade et al. 1990; Getti et al. 2008; Hsiao et al. 2011). In order to identify whether such model is suitable to represent parasite spread, differentiated THP-1 macrophages were infected with $L$. aethiopica (L8G) and L. mexicana (M5G) promastigotes. The percentage of cells harbouring GFP expressing parasites during early (24 and $48 \mathrm{~h}$ ) and late (72 and $96 \mathrm{~h}$ ) stages of infection was quantified via flow cytometry.

After $24 \mathrm{~h}, 57 \cdot 1 \pm 0 \cdot 4$ and $57 \pm 9 \cdot 1 \%$ of cells were infected with L. aethiopica and L. mexicana, 
A

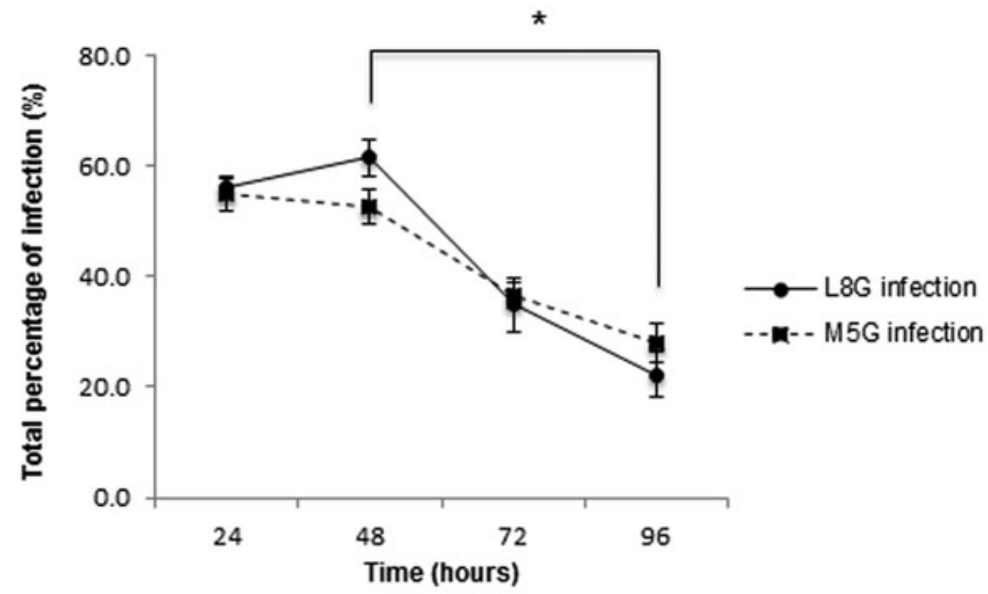

B

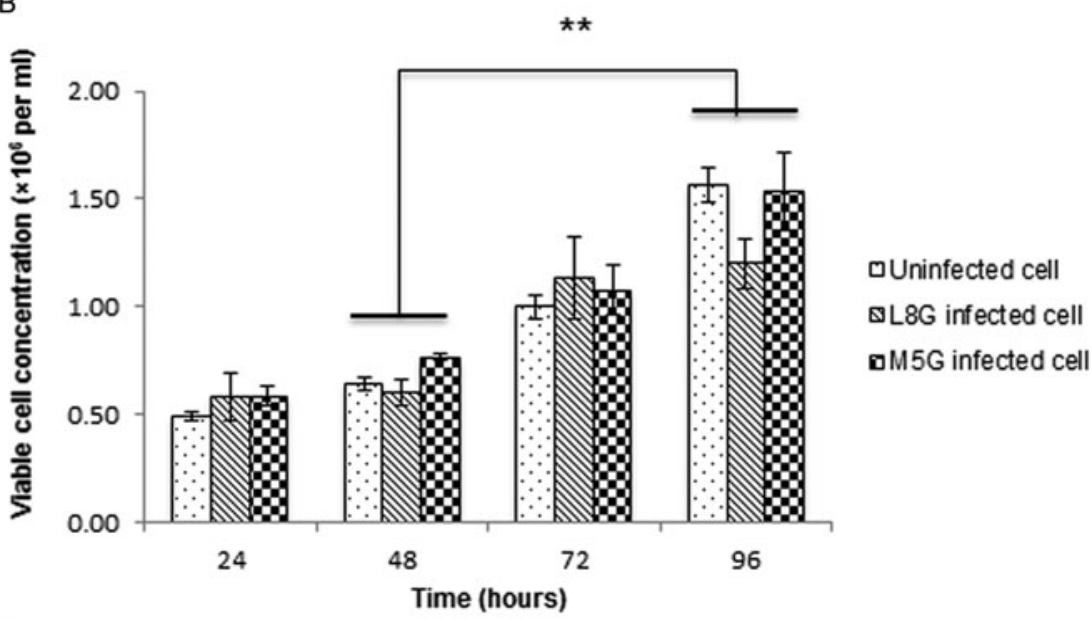

C
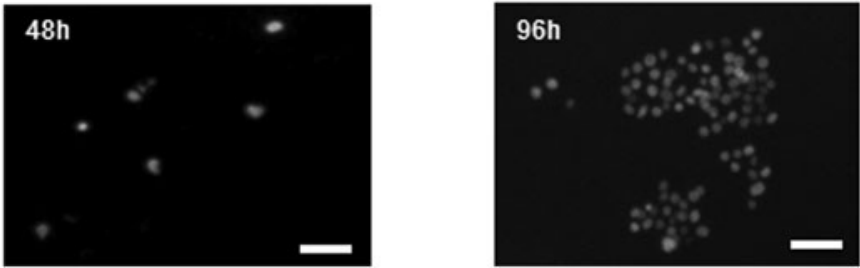

Fig. 1. Infection of THP-1 macrophage with Leishmania promastigotes. (A) Differentiated THP-1 macrophages were infected with two promastigote species, L. aethiopica and L. mexicana at $10: 1$ ratio (parasites to cell ratio). The percentage of infection was determined through flow cytometry at $24,48,72$ and $96 \mathrm{~h}$ of incubation. Data indicate mean percentage of infection \pm s.E.M. from three independent experiments. (B) Uninfected, L. aethiopica infected and L. mexicana infected cells were stained with trypan blue solution at $24,48,72$ and $96 \mathrm{~h}$ of incubation. Data indicate mean concentration of viable cells \pm s.E.M. from three independent experiments. (C) Fluorescence microscopic imaging of DAPI positive nuclei of uninfected cells from 48 and $96 \mathrm{~h}$. Bar $=50 \mu \mathrm{m}$. L8G, L. aethiopica; M5G, L. mexicana; ${ }^{*} P<0.05$ and ${ }^{*} * P<0.01$.

respectively (Fig. 1A). This level of infection for both species was maintained till $48 \mathrm{~h}$ of continuous incubation. However, no spreading was observed as, there was a significant decrease $(P<0.05)$ in the percentage of $L$. aethiopica and $L$. mexicana infected cells from 48 to $96 \mathrm{~h}$ (Fig. 1A). To clarify the cause of this reduction, cell viability was quantified and showed a 2 -fold increase in the concentration of viable cells from 48 to $96 \mathrm{~h}$ in both uninfected and infected cells suggesting that the cells had resumed replication (Fig. 1B and C).
Effect of apoptotic induction on the rate of Leishmania infection

Induction of host cell apoptosis has been reported to promote cell-to-cell dissemination of L. amazonensis during mice macrophage infection in vitro (Real et al. 2014). Therefore, we investigated whether apoptotic induction could also support L. aethiopica and L. mexicana spreading within the infection model based on THP-1 macrophage. Camptothecin is a quinoline alkaloid, which binds to topoisomerase I and DNA complex, stabilising it and causing DNA 


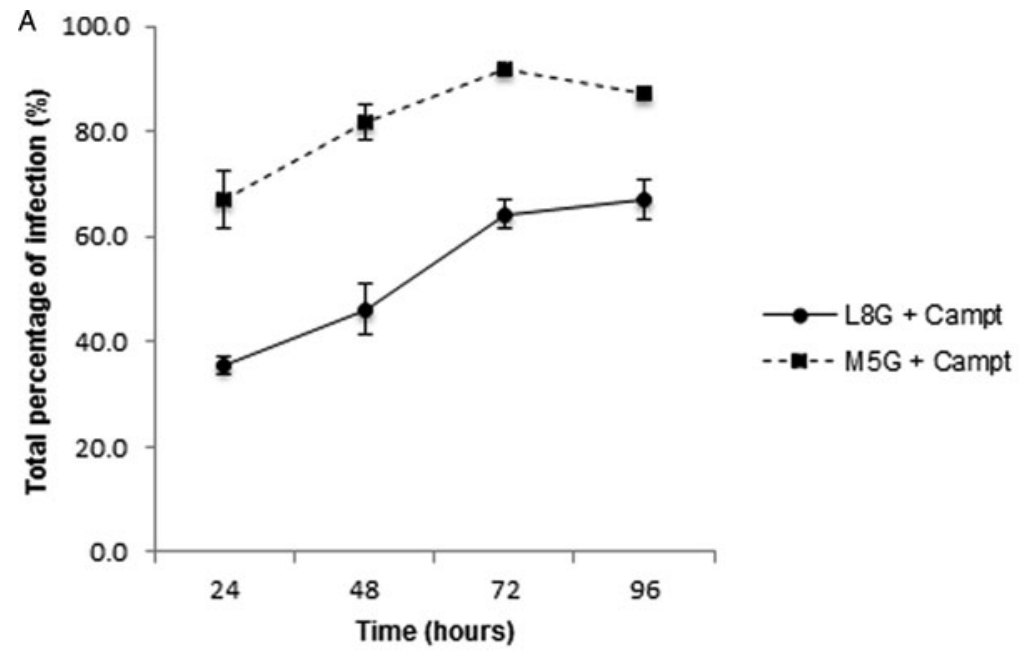

B

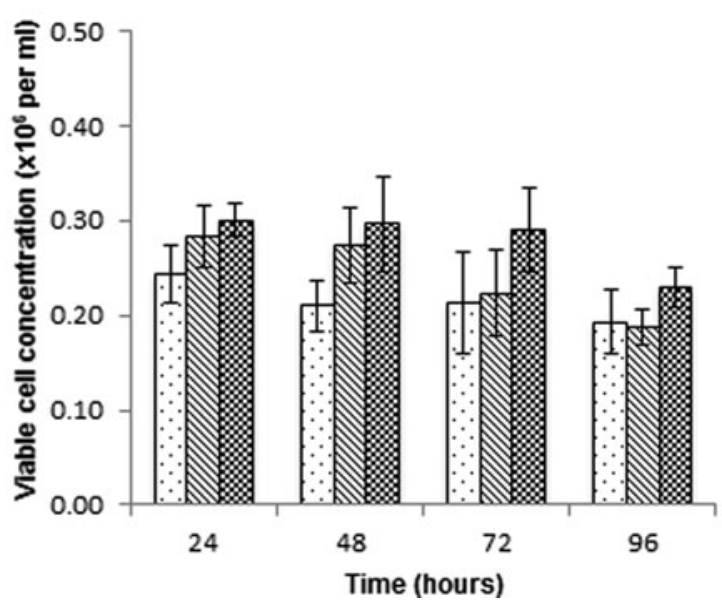

\author{
घUninfected cell + Campt \\ هL8G infected cell + Campt \\ M5G infected cell + Campt
}

Fig. 2. Effect of apoptotic induction on the rate of Leishmania infection. (A) Differentiated THP-1 macrophages were infected with L. aethiopica and L. mexicana promastigotes at $10: 1$ ratio (parasite to cell ratio) and treated with Campt to induce apoptosis. Excess Campt was washed away from culture medium at $24 \mathrm{~h}$ after L. aethiopica and $5 \mathrm{~h}$ after L. mexicana infection. The percentage of infection was determined through flow cytometry at $24,48,72$ and $96 \mathrm{~h}$ of incubation. Data indicate mean percentage of infection \pm s.E.M. from three independent experiments. (B) Campt treated uninfected or infected cells by L. aethiopica and L. mexicana were stained with trypan blue solution at 24, 48, 72 and $96 \mathrm{~h}$ of incubation. Data indicates mean concentration of viable cells \pm s.E.M. from three independent experiments. L8G,

L. aethiopica; M5G, L. mexicana; Campt, Camptothecin; * $P<0.05$.

damage which results in apoptosis. The optimal concentration and length of camptothecin treatment was identified for each infecting species (data not shown). Based on optimised conditions, L. aethiopica and L. mexicana infected cells were treated with camptothecin for 24 and $5 \mathrm{~h}$ respectively, after infection and the percentage of infected cells was detected over a period of $96 \mathrm{~h}$.

At $24 \mathrm{~h}, 35 \cdot 4 \pm 2$ and $67 \cdot 1 \pm 5 \cdot 4 \%$ of cells were infected by $L$. aethiopica and L. mexicana, respectively, in the presence of camptothecin treatment (Fig. 2A). Interestingly, the treatment caused a significant increase $(P<0 \cdot 05)$ in the percentage of infection for both species, reaching a maximum of $64 \cdot 1 \pm$ $3 \%$ for $L$. aethiopica and $92 \pm 1 \cdot 2 \%$ for $L$. mexicana within $72 \mathrm{~h}$ before plateauing at $96 \mathrm{~h}$ (Fig. 2A). This indicated that camptothecin treatment did not affect intracellular parasites viability. The concentration of viable cells was similar from 24 to $96 \mathrm{~h}$ infection, but significantly lower than the non-treated samples (Figs $1 \mathrm{~B}$ and $2 \mathrm{~B}$ ).

\section{Infection of differentiated THP-1 macrophages with} L. aethiopica and L. mexicana infected cells

Despite the low concentration of viable cells in camptothecin treated L. aethiopica and L. mexicana-infected samples (compared with nontreated), a heavy proportion of infected cells were obtained at 72 and $96 \mathrm{~h}$. To determine which of the infected culture, 72 or $96 \mathrm{~h}$, would be suitable as a donor population, the long-term effect of camptothecin treatment on THP-1 cells was determined. Annexin $\mathrm{V}$ assay showed no significant difference between the percentage of apoptosis of L. aethiopica and $L$. mexicana infected cells with and without $72 \mathrm{~h}$ 


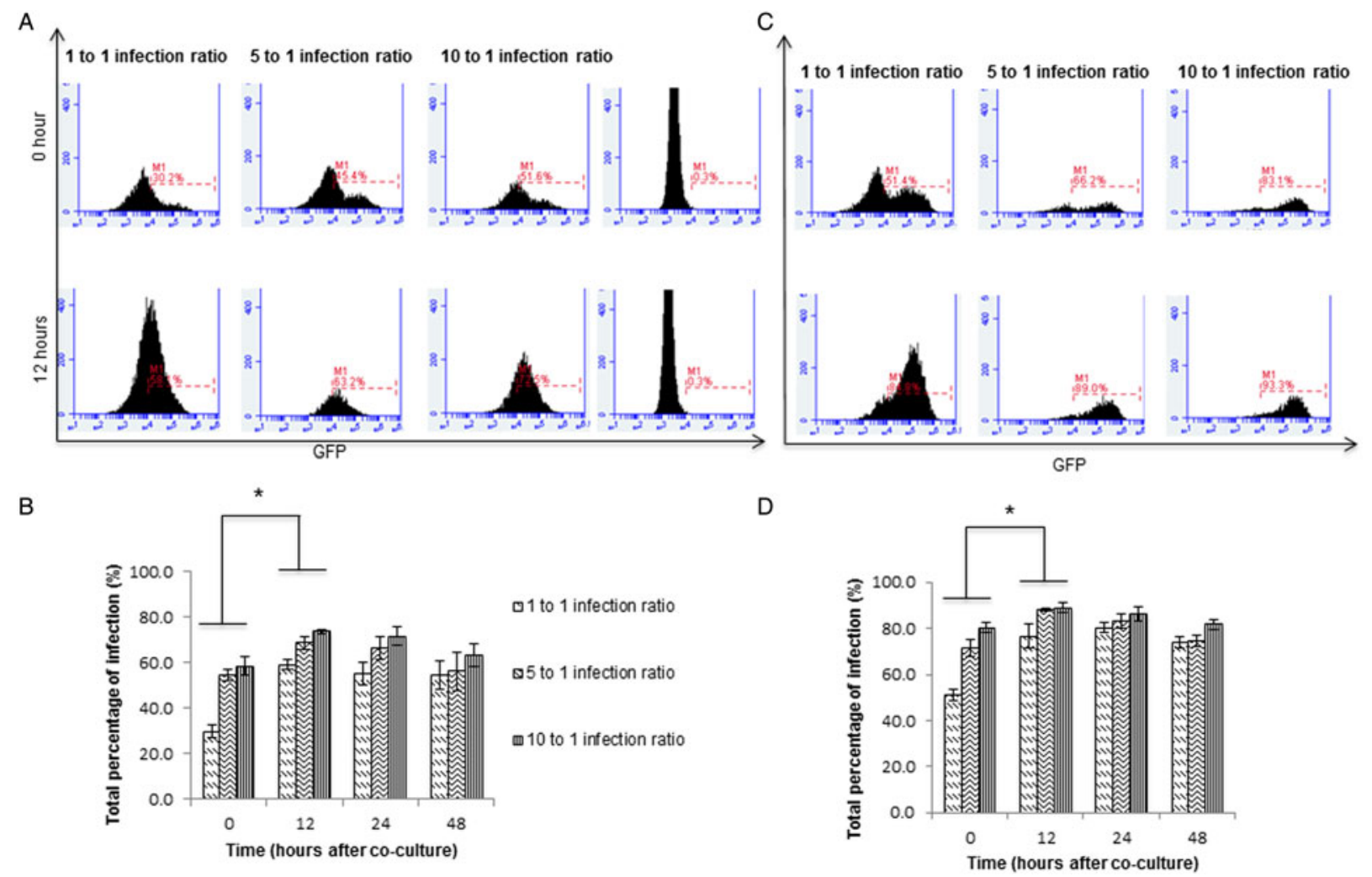

Fig. 3. Infection of THP-1 macrophages with Leishmania infected cells. (A-D) After $72 \mathrm{~h}$ of infection, apoptotic induced L. aethiopica and L. mexicana infected cells were co-cultured with newly differentiated THP-1 macrophage at different infection ratios, $1: 1,5: 1$ and $10: 1$ (infected to uninfected cell ratio). (A) Flow cytometry histogram plot representative of L. aethiopica infected cells 'M1' at 0 and $12 \mathrm{~h}$ after co-culture. (B) Data indicate mean percentage of L. aethiopica infection \pm s.E.M. from three independent experiments at $0,12,24$ and $48 \mathrm{~h}$ after co-culture. (C) Flow cytometry histogram plot representative of L. mexicana infected cells 'M1' at 0 and $12 \mathrm{~h}$ after co-culture. (D) Data indicate mean percentage of L. mexicana infection \pm s.E.M. from three independent experiments at $0,12,24$ and $48 \mathrm{~h}$ after co-culture. L8G, $L$. aethiopica; M5G, L. mexicana; *P<0.05.

camptothecin treatment (Supplementary Fig. S1A). In contrast, the treated L. aethiopica and L. mexicana-infected cells displayed a significantly higher percentage of apoptosis than the non-treated culture at $96 \mathrm{~h}$ (Supplementary Fig. S1B). Therefore, to remove any experimental bias due to camptothecin treatment, the $72 \mathrm{~h}$ infected population $(64 \cdot 1 \pm 3$ and $92 \pm 1 \cdot 2 \%$ ) was used as a donor to infect newly differentiated THP-1 macrophages. Different infection ratios were tested $(1: 1,5: 1$ and $10: 1$ infected to uninfected cell ratio) and the course of infection was detected at $0,12,24$ and $48 \mathrm{~h}$ after co-culture through flow cytometry.

Immediately $(0 \mathrm{~h})$ after co-culture between infected and uninfected cells, a dilution effect on the percentage of infection for both $L$. aethiopica and L. mexicana was detected as expected (Fig. 3A and $\mathrm{C}$ ). Following $12 \mathrm{~h}$ of co-culture, the percentage of $L$. aethiopica-infected cells almost doubled from $35 \pm 2 \cdot 3$ to $60 \pm 2 \cdot 1 \%$ for $1: 1$ and significantly increased $(P<0 \cdot 05)$ for $5: 1 \quad(54 \pm 2 \cdot 4$ to $69 \pm 3 \%)$ and $10: 1(58 \pm 4 \cdot 1$ to $74 \pm 0 \cdot 4 \%)$ infection ratios (Fig. 3B). This was also observed during coculture with $L$. mexicana-infected cells; $51 \pm 2 \cdot 3$ to
$76 \pm 5 \cdot 2 \%$ for $1: 1,71 \pm 3 \cdot 3$ to $88 \cdot 2 \pm 1 \%$ for $5: 1$ and $80 \pm 2 \cdot 1$ to $90 \pm 2 \cdot 3 \%$ for $10: 1$ infection ratios (Fig. 3D). However, such effects were not detected in the negative control, comprising non-treated infected cells co-cultured with uninfected cells. The trypan blue assay showed no significant difference in the concentration of viable cells between 0 and $12 \mathrm{~h}$ of co-culture (Fig. 4A and B). This suggested that the infection increase from 0 to $12 \mathrm{~h}$ time frame was due to spreading of $L$. aethiopica and L. mexicana amastigotes from the $72 \mathrm{~h}$ donor infected cells to healthy cells and not caused by the reduction of uninfected cells. No further increase in the percentage of L. aethiopica and L. mexicanainfected cells was detected from 12 to $24 \mathrm{~h}$ after coculture (Fig. 3B and D).

Overall, the above results provided quantitative evidence supporting the hypothesis that L. aethiopica and L. mexicana amastigotes spreading takes place from 0 to $12 \mathrm{~h}$ after co-culture. This in vitro spreading model provides an initial platform to address which mode of exit is utilized by both species of Leishmania in order to transfer from cellto-cell. 


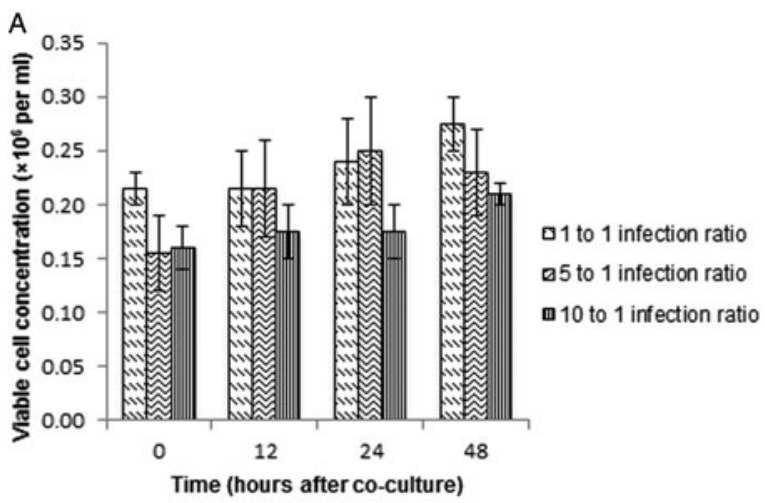

selectively engulf $L$. aethiopica and L. mexicana via recognition of parasite or host associated markers present in the donor cell.

\section{DISCUSSION}

The development of leishmaniasis and associated pathogenesis is dependent on Leishmania amastigotes ability to successfully disseminate to uninfected macrophages. Despite the importance of this virulence mechanism on the outcome of infection, a lack of a reliable infection model representative of parasite-host interaction has led to a major gap in knowledge pertaining to the understanding of Leishmania spreading in human macrophages. Here, we have reported the development of a coculture-based experiment, which models cell-tocell dissemination of L. aethiopica and L. mexicana parasites. The spreading of parasites was evaluated by monitoring the total percentage of infection as well as cell viability before and after co-culture. This approach, along with live imaging, not only confirmed spreading but also supported a previous observation that some Leishmania species can exit host cells in a non-lytic process.

During natural Leishmania infection, macroFig. 4. Measurement of viable cell concentration during infection with Leishmania infected cells. L. aethiopica infected co-culture (A) and L. mexicana-infected co-culture (B) cells at different infection ratios were stained with trypan blue solution at $0,12,24$ and $48 \mathrm{~h}$ after co-culture. Data indicate mean concentration of viable cells \pm s.E.M. from three independent experiments. L8G, L. aethiopica; M5G, L. mexicana .

\section{Live cell imaging of intracellular L. aethiopica and L. mexicana amastigotes spreading}

During cell-to-cell infection spreading, intracellular pathogens within the donor cells can trigger either a lytic or a non-lytic release (Friedrich et al. 2012). To verify whether such a process might occur during $L$. aethiopica and L. mexicana release, time lapse microscopy was performed.

Initially, differentiated THP-1 macrophages (prestained with CellMask ${ }^{\mathrm{TM}}$ orange plasma membrane stain) were co-cultured with $L$. aethiopica and/or $L$. mexicana infected cells and imaged over $12 \mathrm{~h}$. From $8 \mathrm{~h}$, L. aethiopica amastigotes started migrating towards the peripheral membrane of the donor cells, before completely transferring into the already infected recipient stained cells within $10 \mathrm{~min}$ of recording (Fig. 5A, Supplementary Video 1). This phenomenon was also visualized in L. mexicanainfected co-culture whereby after $3 \mathrm{~h}$, an amastigote appeared to be extruded into the recipient stained cells (Fig. 5B, Supplementary Video 2). Since donor cell (in both scenarios) remained intact during amastigote transfer, it is evident that Leishmania spread can take place via a non-lytic mode in human cells. It remains unclear whether recipient macrophages phages are the definitive human cells for parasite growth and replication. To model this host-parasite interaction in vitro, many investigators have used either macrophage-like cell lines or human blood derived macrophages (Ogunkolade et al. 1990; Lisi et al. 2005; Ruhland et al. 2007). Since primary blood derived macrophages do not support replication and spreading of amastigotes (Hsiao et al. 2011), cell lines were selected in our in vitro model. The choice of human THP-1 is further supported by the fact that they are non-adherent, which makes them ideal for flow cytometry studies. The use of adherent cell lines requires detachment through trypsin treatment that might damage cell membranes causing subsequent parasite release, leading to inconsistent data (Huang et al. 2010). Direct infection of differentiated THP-1 macrophages with promastigotes was not sufficient to detect spreading as the effect of retinoic acid started to subside after $48 \mathrm{~h}$. At this point, THP-1 replication resumed causing the percentage of infected cells to decrease, eventually making the model unsuitable for further studies on parasitic spreading.

Treatment with an apoptotic inducer immediately after infection was successful in overcoming the above challenge and producing a population containing a high percentage of infected cells. Induction of host cell apoptosis is considered as one of the major routes for the spreading of many virulent pathogens including, Mycobacterium tuberculosis, Chlamydia and adenovirus (Mi et al. 2001; Byrne and Ojcius, 2004; Aguilo et al. 2013). The 

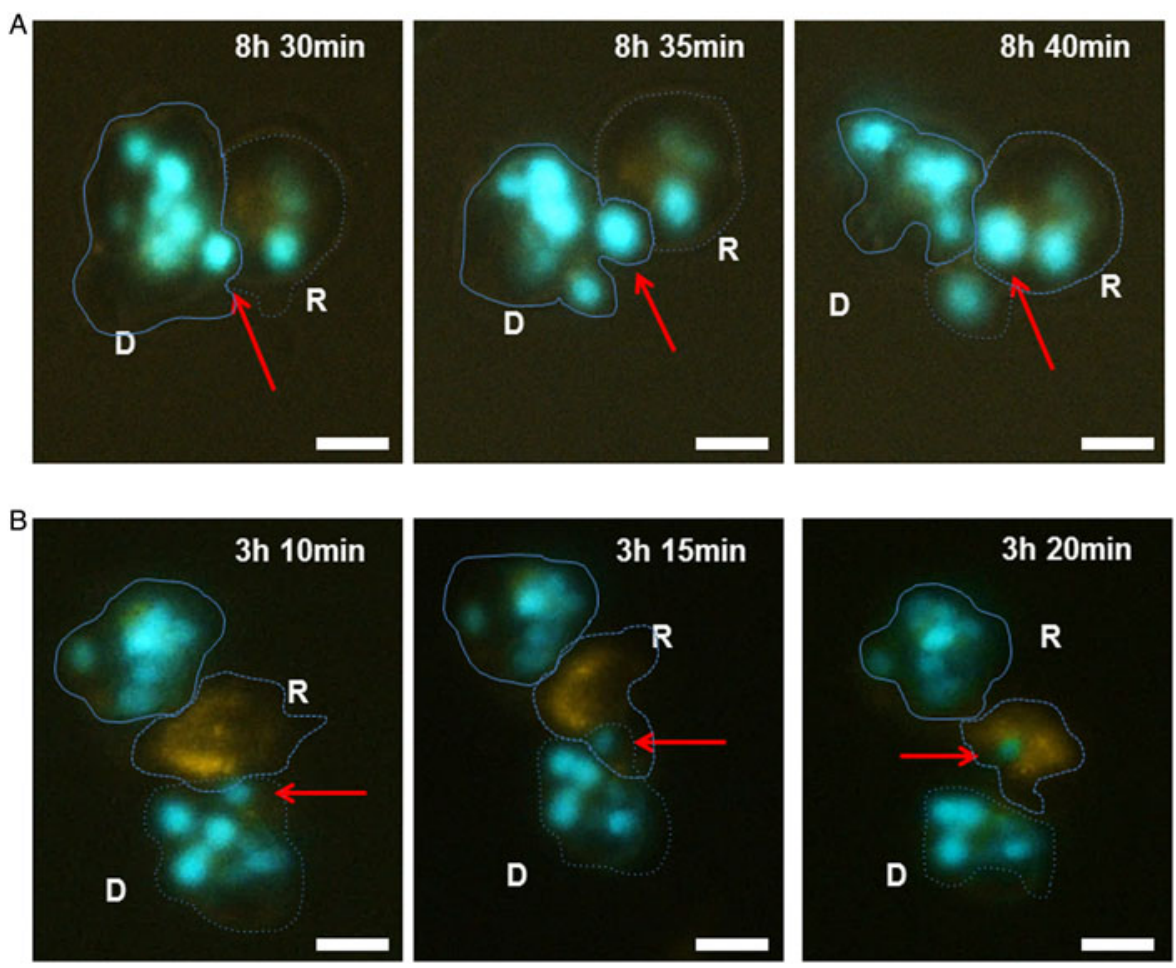

Fig. 5. Cell-to-cell spreading of Leishmania amastigotes. Freshly differentiated THP-1 cells acting as recipient uninfected cells (labelled as R), were pre-stained with CellMask ${ }^{\mathrm{TM}}$ orange plasma membrane dye. The recipient cells were then cocultured with L. aethiopica (A) and L. mexicana (B) infected cells, which act as donor cells (labelled as D). The time of acquisition is represented by hours $(\mathrm{h})$ and minutes $(\mathrm{min})$. Image acquisition started at 1 hour after co-culture. Bar $=25 \mu \mathrm{m}$.

link between apoptosis of host macrophages and Leishmania infection is complex. Parasites have shown the ability to protect host cells from apoptotic induction at early stages of infection (Moore and Matlashewski, 1994; Akarid et al. 2004; Lisi et al. 2005; Ruhland et al. 2007; Donovan et al. 2009) but a link between apoptosis and spreading has also been reported (Getti et al. 2008; Real et al. 2014; DaMata et al. 2015). Based on either set of evidence, apoptotic induction was expected to produce a large proportion of infected macrophages, which it did over a period of 72 and $96 \mathrm{~h}$. Therefore, to mimic a natural form of parasitic dissemination, a high proportion of L. aethiopica and L. mexicana-infected cells from either 72 or $96 \mathrm{~h}$ were chosen as the donor population before co-culturing with uninfected freshly differentiated THP-1 macrophages. This form of cell-to-cell infection has been successfully utilized in studies of other infectious agents such as HIV-1 (Groppelli et al. 2015), Mycobacterium (Hagedorn et al. 2009) and L. amazonensis (Real et al. 2014). However, prior to carrying out such an experiment, it was very important to select a suitable time point for co-culture. Specifically, a time during which the effect of camptothecin treatment on infected cells viability had subsided. Careful analysis of early apoptotic markers showed that after $72 \mathrm{~h}$ infection the treated population of infected cells expressed a similar percentage of apoptosis to the untreated, infected population. The time point of $72 \mathrm{~h}$ was therefore identified as an appropriate point of coculture.

Results from flow cytometry and trypan blue assay confirmed that the increase in percentage of infection from 1 to $12 \mathrm{~h}$ was due to intracellular amastigote dissemination and not caused by a decrease in viable cell number. Moreover, microscopy clearly showed parasites spreading during which both species were filmed exiting the host cell via nonlytic mode. This corresponds with a previous report by Real et al. (2014), where the authors observed that L. amazonensis amastigotes were surrounded by a host membrane during their egress from mice macrophages. It cannot be excluded that amastigotes could also cause macrophages to rupture and once released be picked up by neighbouring macrophages as extracellular parasites. Nevertheless, our findings suggest that L. aethiopica and L. mexicana parasites have developed a mechanism whereby they do not need to burst the host cells to spread to uninfected macrophages. Parasites can be slowly released from the host cells without damaging them. Such mechanism would explain the silent phase of amastigote spreading within the host body, when no inflammatory response is detected (Belkaid et al. 2000). This mechanism in L. aethiopica and L. mexicana could be regulated via apoptotic pathways, or could involve an alternative form of induction of membrane budding and/or actin based extrusion, which needs to be investigated further. 
In summary, for the very first time, we have successfully developed an in vitro model representative of Leishmania spreading in human macrophages. This was achieved in two steps. First, a population containing a high percentage of infected cells was obtained via camptothecin treatment of infected cells. This population was then used as a donor to infect a new and healthy population of differentiated THP-1 macrophages. This form of co-culture experiment allowed us to identify the timeframe for the inter-cellular dissemination of L. aethiopica and L. mexicana spreading to uninfected cells. Live microscopy revealed non-lytic release of amastigotes from cell-to-cell and provided confirmation of a strong link with quantitative flow cytometry data on infection spreading. Following the establishment of the above model, the next step will be to functionally characterize virulence factors associated with amastigote spreading and decipher the cellular and molecular mechanism by which Leishmania exit the host cells in non-lytic mode, an issue neglected in the study of these parasites.

\section{SUPPLEMENTARY MATERIAL}

The supplementary material for this article can be found at https://doi.org/10.1017/S0031182017001366

\section{ACKNOWLEDGEMENTS}

We thank Samantha Ingram, Richard Anthony and Rachael Nice for expert technical assistance and Stuart Connop for proofreading the manuscript. The work was funded by the University of Greenwich VC PhD studentship.

\section{REFERENCES}

Aguilo, N., Marinova, D., Martin, C. and Pardo, J. (2013). ESX-1 induced apoptosis during mycobacterial infection: to be or not be, that is the question. Frontiers in Cellular and Infection Microbiology 3, 88.

Akarid, K., Arnoult, D., Micic-Polianski, J., Sif, J., Estaquier, J. and Claude Ameisen, J. (2004). Leishmania major mediated prevention of programmed cell death induction in infected macrophages is associated with the repression of mitochondrial release of cytochrome c. Fournal of Leukocyte Biology 76, 95-103.

Alvar, J., Vélez, I. D., Bern, C., Herrero, M., Desjeux, P., Cano, J., Jannin, J. and de Boer, M. (2012). Leishmaniasis worldwide and global estimates of its incidence. PLOS ONE 7, pp: e35671.

Ashida, H., Mimuro, H., Ogawa, M., Kobayashi, T., Sanada, T., Kim, M. and Sasakawa, C. (2011). Cell death and infection: a doubleedged sword for host and pathogen survival. Fournal of Cell Biology 195, 931-942.

Belkaid, Y., Mendez, S., Lira, R., Kadambi, N., Milon, G. and Sacks, D. (2000). A natural model of Leishmania major infection reveals a prolonged silent phase of parasite amplification in the skin before the onset of lesion formation and immunity. Fournal of Immunology 165, 969-977. Bifeld, E. and Clos, J. (2015). The genetics of Leishmania virulence. Medical Microbiology and Immunology 204, 619-634.

Byrne, G. I. and Ojcius, D. M. (2004). Chlamydia and apoptosis: life and death decisions of an intracellular pathogen. Nature Reviews Microbiology 2, 802-808.

Cameron, P., McGachy, A., Anderson, M., Paul, A., Coombs, G. H., Mottram, J. C., Alexander, J. and Plevin, R. (2004). Inhibition of Lipopolysaccharide induced macrophage IL-12 production by Leishmania mexicana amastigotes: the role of cysteine peptidases and the NF-kB signaling pathway. Fournal of Immunology 173, 3297-3304.

DaMata, J.P., Mendes, B.P., Maciel-Lima, K., Menezes, C. A., Dutra, W. O., Sousa, L.P. and Horta, M. F. (2015). Distinct macrophages fates after in vitro infection with different species of Leishmania: induction of apoptosis of Leishmania (Leishmania) amazonensis, but not by Leishmania (Viannia) guyanensis. PLOS ONE 10, pp: e0141196.

De Souza, S., Lang, T., Prina, E. and Hellio, R. (1995). Antoine JC. Intracellular Leishmania amazonensis amastigotes internalize and degrade MHC class II molecules of their host cells. Fournal of Cell Science 108, 3219-3231.

Dominguez, M., Moreno, I., Aizpurua, C. and Torano, A. (2003). Early mechanisms of Leishmania infection in human blood. Microbes and Infection 5, 507-513.

Donovan, M. J., Maciuba, B. Z., Mahan, C. E. and McDowell, M. A. (2009). Leishmania infection inhibits cycloheximide induced macrophage apoptosis in a strain dependent manner. Experimental Parasitology 123, 58-64 Friedrich, N., Hagedorn, M., Soldati-Favre, D. and Soldati, T. (2012). Prison break: pathogens' strategies to egress from host cells. Microbiology and Molecular Biology Reviews 76, 707-720.

Getti, G., Cheke, R. A. and Humber, D. P. (2008). Induction of apoptosis in host cells: a survival mechanism for Leishmania parasites? Parasitology 135, 1391-1399.

Gomez, R. and Oliveira, F. (2012). The immune response to sand fly salivary proteins and its influence on leishmania immunity. Frontiers in Immunology 3, pp 110.

Gregory, D. J., Godbout, M., Contreras, I., Forget, G. and Olivier, M. (2008). A novel form of NF-kB is induced by Leishmania infection: involvement in macrophage gene expression. European fournal of Immunology 38, 1071-1081.

Groppelli, E., Starling, S. and Jolly, C. (2015). Contact-induced mitochondrial polarization supports HIV-1 virological synapse formation. Fournal of Virology 89, 14-24.

Hagedorn, M., Rohde, K. H., Russell, D. G. and Soldati, T. (2009). Infection by tubercular mycobacteria is spread by nonlytic ejection from their amoeba hosts. Science 323, 1729-1733.

Hsiao, C. H. C., Ueno, N., Shao, J. Q., Schroeder, K. R., Moore, K. C., Donelson, J. E. and Wilson, M. E. (2011). The effects of macrophage source on the mechanism of phagocytosis and intracellular survival of Leishmania. Microbes and Infection 13, 1033-1044.

Huang, H. L., Hsing, H.W., Lai, T.C., Chen, Y.W., Lee, T.R., Chan, H. T., Lyu, P. C., Wu, C. L., Lu, Y.C., Lin, S. T., Lin, C. W., Lai, C. H., Chang, H. T., Chou, H.C. and Chan, H. L. (2010). Trypsin induced proteome alteration during cell subculture in mammalian cells. Fournal of Biomedical Science 17, pp 36.

Kedzierski, L. (2010). Leishmaniasis Vaccine: where are we today? Fournal of Global Infectious Diseases 2, 177-185.

Kimblin, N., Peters, N., Debrabant, A., Secundino, N., Egen, J., Lawyer, P., Fay, P., Kamhawi, S. and Sacks, D. (2008). Quantification of the infectious dose of Leishmania major transmitted to the skin by single sand flies. PNAS 105, 10125-10130.

Lisi, S., Sisto, M., Acquafredda, A., Spinelli, R., Schiavone, M. A., Mitolo, V., Brandonisio, O. and Panaro, M. A. (2005). Infection with Leishmania infantum inhibits actinomycin D induced apoptosis of human monocytic cell line U-937. Fournal of Eukaryotic Microbiology 52, 211-217. Lodge, R. and Descoteaux, A. (2005). Modulation of phagolysosome biogenesis by the lipophosphoglycan of Leishmania. Clinical Immunology 114, 174-181.

Mi, J., Li, Z., Ni, S., Steinwaerder, D. and Liber, A. (2001). Induced apoptosis supports spread of adenovirus vectors in tumours. Human Gene Therapy 12, 1343-1352.

Mißlitz, A., Mottram, J. C., Overath, P. and Aebischer, T. (2000). Targeted integration into a rRNA locus results in uniform and high level expression of transgenes in Leishmania amastigotes. Molecular and Biochemical Parasitology 107, 251-261.

Moore, K. J. and Matlashewski, G. (1994). Intracellular infection by Leishmania donovani inhibits macrophage apoptosis. Fournal of Immunology 152, 2930-2937.

Ogunkolade, B.W., Colomb-Valet, I., Monjour, L., RhodesFeuillette, A., Abita, J.P. and Froomel, D. (1990). Interactions between the human monocytic leukaemia THP-1 cell line and ld and New World species of Leishmania. Acta Tropica 47, 171-176.

Patel, A. P., Deacon, A. and Getti, G. (2014). Development and validation of four Leishmania species constitutively expressing GFP protein. A model for drug discovery and disease pathogenesis studies. Parasitology 141, 501-510.

Real, F., Florentina, P.T.V., Reis, L. C., Ramos-Sanchez, E. M., Veras, P.S. T., Goto, H. and Mortara, R. A. (2014). Cell-to-cell transfer of Leishmania amazonensis amastigotes is mediated by immunomodulatory LAMP-rich parasitophorous extrusions. Cellular Microbiology 16, 15491564 . 
Richardson, S.C., Wallom, K. L., Ferguson, E. L., Deacon, S.P., Davies, M. W., Powell, A. J., Piper, R. C. and Duncan, R. (2008).

The use of fluorescence microscopy to define polymer localisation to the late endocytic compartments in cells that are targets for drug delivery. Fournal of Controlled Release 127, 1-11.

Rogers, M. E., IIg, T., Nikolaev, A. V., Ferguson, M. A. and Bates, P. A. (2004). Transmission of cutaneous leishmaniasis by sand flies is enhanced by regurgitation of fPPG. Nature 430, 463-467.
Ruhland, A., Leal, N. and Kima, P. E. (2007). Leishmania promastigotes activate PI3K/AKT signaling to confer host cell resistance to apoptosis. Cellular Microbiology 9, 84-96.

Seifert, K., Escobar, P. and Croft, S. L. (2010). In vitro activity of anti leishmanial drugs against Leishmania donovani is host cell dependent. fournal of Antimicrobial Chemotherapy 65, 508-511.

Stamm, L. V. (2016). Human migration and leishmaniasis - on the move. DAMA Dermatology 152, 373-374. 